\title{
COMPLEX TREATMENT OF PURULENT WOUNDS WITH THE USE OF HIGH-PRESSURE AERODISPERSE MIXTURE
}

DOI: 10.36740/WLek202005110

\author{
Volodymyr 0. Shaprynskyi, Stepan S. Skalskyi, Yevhen V. Shaprynskyi, Andriy V. Verba, Viktor M. Makarov \\ DEPARTMENT OF SURGERY №1, NATIONAL PIROGOV MEMORIAL MEDICAL UNIVERSITY, VINNYTSIA, UKRAINE
}

\begin{abstract}
The aim: To investigate the effectiveness of complex treatment of purulent wounds with the use of high pressure aerodispersed mixture of antibacterial drug using sorbents and correction of hydrogen index.

Materials and methods: In total, 54 patients were treated, out of which there were 26 patients with purulent wounds in the main group and 28 patients in the control group. Patients in the main group were treated according to the developed method, patients in the control group were treated with traditional antiseptics and water soluble ointments. Results: Within patients of the main group, there was a decrease in the duration of inflammatory process, decrease in the quantity of microorganisms in the wound; slight changes in the sensitivity of the microorganisms to antibacterial preparations, compared with the control group, which showed a high percentage of development of resistant strains; decreased edema and secretion from wounds, the early appearance of granulation.

Conclusions: The use of the proposed approach reduces the microbial burden on the wound, accelerates its purification from purulent-necrotic tissues and reduces the duration of the inflammatory process due to the presence of the microbial factor.
\end{abstract}

KEY WORDS: wound infection, topical antimicrobial agents, wound-milieu

\section{INTRODUCTION}

At present, according to various estimates, from $40 \%$ to $70 \%$ of patients go to medical surgeries with purulent surgical soft tissue infection [1]. Skin and soft tissue infections (SSTIs) cover a range of pathological conditions of the skin and subcutaneous tissue, and affect the whole body [2]. Recently, the US FDA introduced a new definition of Acute Bacterial Skin and Skin Disease (ABSSSI) to more accurately detect complicated soft tissue infections for registration tests. ABSSSI include cellulite / bestial, wound infections and large skin abscesses. Thus, ABSSSI is defined as a bacterial infection of the skin with an area of $\geq 75 \mathrm{~cm}$ 2 (size measured as redness, edema, or consolidation) US Food and Drug Administration. Guidance for industry. Acute bacterial skin and skin structure infections: developing drugs for treatment. In 2015, the Worldwide Society for Emergency Surgery (WSES) published its guidelines for the management of SSTI by proposing a new definition that divides SSTIs into three main groups: infection at the site of surgery, ie., surgical site infections (SSIs), necrotizing SSTIs and non-necrotizaing SSTIs. SSIs are further divided into two subgroups: incisional and organ and organ/spatial. Incisional SSIs are further divided into superficial (skin and subcutaneous tissue) and deep (deep soft-tissue muscle and fascia). Organ and organ/space infections are not truly soft-tissue infections. Non-necrotizing SSTIs including erysipelas, impetigo, folliculitis, simple abscess [3]. In 2004, around 1.3 million patients were hospitalized for SSTIs, including as a result of phlegmon (52.7\%), local surgical infection (15.8\%), diabetic foot (15.3\%), bedsores (12\%). In Ukraine, the incidence of disease in SSTIs ranges from 480 to 700 thousand patients per year [4]. A slightly different meaning is the term used in English literature - surgical wounds that are healed by secondary intensity (SWHSI). It includes actually primary wounds healed by secondary tension (for example, after removal of the pilonoid cyst) and wounds in which the healing process is impaired by the primary tension, including due to infection. The amount of SWHSI is about $28 \%$ of all acute healed wounds [5]. For example, the SWHSI prevalence in the UK is 0.41 per 1,000 population [6], the spent amount is about 116 million pounds only for bandages, not including maintenance costs. The prevalence of SSI was estimated at about $5 \%$ of all operations in the UK and Ireland [7]. Open wounds, especially those that are healed with secondary tension, are an ideal environment for microbial colonization, although this does not necessarily lead to festering, there is no clear boundary under the Australian Wound Management Association (AWMA) for critical bacterial contamination. At the same time, according to the consensus of the World Union of Wound Healing Societies (WUWHS), an increase in bacterial wound contamination leads to a decrease in healing rate.

\section{THE AIM}

The aim: to investigate the effectiveness of complex treatment of purulent wounds with the use of high pressure 
aerodisperse mixture of antibacterial drug using sorbents and correction of hydrogen index. To do this, the following tasks should be solved:

1. Experimentally determine the antibacterial effectiveness of the local controlled elevated pressure of the aerosol dispenser mixture of medicinal drugs.

2. To develop a complex algorithm for treatment of purulent wounds using the indicated method of treatment. 3. To study the dynamics of cytological, microbiological changes of wound healing using the developed method and evaluate its clinical efficiency.

\section{MATERIALS AND METHODS}

Biomedical Ethics Commission of Vinnytsya National Pirogov Memorial Medical University on observance of moral and legal rules in conducting medical research has established that clinical research were carried out in compliance with the main provisions of GCP (1996), Council of Europe Convention for Human Rights and Biomedicine (1997), World Medical Association Declaration of Helsinki on ethical principles for medical research involving human subjects (1964-2000) and Order of Ministry of Health of Ukraine № 281 of November 1, 2000.

To solve the first task, a device was designed that modeled the closed space above the wound (Pat. 128108 Ukraina, MPK51 A61K9/12, A61K35/74, A61J3/00). Antibacterial aerosol sprayed under pressure of $18-20 \mathrm{~mm} \mathrm{Hg}$. over open pots of Petri seeded with biomaterial patient's wounds, with the definition of the minimum amount of drug, which completely suppresses the growth of CSO. The one-time dose of the medicinal product obtained in this way was subsequently used to spray over the wound. Patients in the main group were treated according to the developed method, which included the formation of an air-tight chamber over the wound with the protection of the surrounding skin with an antibacterial pallicle of the type "Ioban" with simultaneous drainage of the wound. An aerosolized antibacterial mixture was applied to the cavity of the chamber at a pressure of $15-20 \mathrm{~mm} \mathrm{Hg}$. An excess of pressure and fluid from the wound was withdrawn through the drainage tube (Pat. 100923 Ukraina, MPK51 A61M11/02, A61M27/00, A61M1/00). Prior to the procedure, the surface $\mathrm{pH}$ was measured, the wound was coated with a layer of sorbent containing silicon dioxide for 10-15 minutes, after which the latter was washed off with a physiological solution of sodium chloride and adjusted to a slightly acidic $\mathrm{pH}$ ( $\mathrm{pH}$ 6.5-6.8), which is optimal for healing and the action of the antibacterial drug. Correction of $\mathrm{pH}$ to the alkaline environment was carried out by a solution of Ringer-lactate (or soda-buffer). An antibiotic solution of 5-7 ml was prepared ex tempore, which was sprayed with an average particle size of the active substance $2.73 \mu \mathrm{m}$.

After the procedure, an air-tight chamber was removed, the wound was covered with a cloth soaked with a physiological solution of sodium chloride. Patients in the comparison group were treated by processing the wound with $3 \%$ hydrogen peroxide, followed by ointment on a water-soluble base, $5 \%$ poidonium iodine, antiseptics based on $0.02 \%$ decamethoxin.

In total, 54 patients were treated, out of which the patients in the main group were 26 patients with purulent wounds, including on the background of diabetes -22 $(84.6 \%)$ patients and 28 patients in the comparison group, among which the patients with diabetes were 24 patients $(85,9 \%)$. Patients of both groups were comparable by age and gender. The $\mathrm{pH}$ was measured by the flat electrode E526BNC and analytical part PH98110 for semi-solid environment (manufacturer Kelilong Electron Co. Ltd). The statistical processing of the results was carried out by the Office Excel 2013 table processor. Evaluation of the results was carried out on 1,3,5 days of treatment with cytological, microbiological and clinical methods.

\section{RESULTS}

Necrotic changes prevailed over 1 day of treatment, fatty detritus against a coarse-fiber intermediate with a small amount of degenerate-altered neutrophils $-70.55 \pm$ $1.73 \%$ in the basic and $73.35 \pm 1.82 \%$ in the comparison group. The number of lymphocytes directly depended on the duration of the inflammatory process and had a wide range of oscillations, from $1 \%$ in acute cases to $40 \%$ in the case of chronic inflammatory process and averaged 7.91 $\pm 0.19 \%$ in the main and $7.85 \pm 0.18 \%$ in the comparison group. In smears, there were isolated monocytes: the main group was $3.55 \pm 0.09 \%$; The comparison group was $2.84 \pm$ $0.07 \%$. Phagocytosis of bacteria, neutrophilic, activity was determined as $13,46 \pm 0,34 \%$ for the main group and 10,12 $\pm 0,25 \%$ for the comparison group. Fibroblasts after 1 day of treatment were observed in extreme cases, histiocytes in the smears were not determined.

Cytological characteristics of the cell composition of the fractures of the wounds of the patients in the main group after 3 days of treatment: neutrophils $79,36 \pm 1,97 \%$, of which degenerative forms $-60,36 \pm 1,48 \%$; lymphocytes $-10.91 \pm 0.27 \%$, monocytes $-5.03 \pm 0.12 \%$; macrophages $3,36 \pm 0,08 \%$, fibroblasts $1,33 \pm 0,33 \%$, percentage of phagocytosis $20,73 \pm 0,51 \%$. Cytological characteristics of the cell composition of the fractures of the wounds of patients in the comparison group after 3 days of treatment: neutrophils $74,48 \pm 1,82 \%$, of which degenerative forms $-70,5 \pm 1,75 \%$; lymphocytes $-14.35 \pm 0.35 \%$; monocytes $-7.86 \pm 0.19 \%$; macrophages $1.89 \pm 0.05 \%, 1.42 \pm 0.03 \%$, phagocytosis activity $15.6 \pm 0.38 \%$. At day 5 of treatment in the cytological picture of the main group, the positive dynamics was maintained as a decrease in the number of polymorphic-nuclear leukocytes $(72.18 \pm 1.77 \%)$, and their degenerative forms $(44.45 \pm 1.08 \%)$, increased activity of phagocytosis ( $24.82 \pm 0.62 \%)$. Single and small mononuclear cells (monocytes) with a tendency to further differentiation $(6.36 \pm 0.16 \%)$ were observed, among which there were giant multicellular and mast cells, and lymphocytes $13.91 \pm 0.34 \%$. The number of fibroblasts $(2.18 \pm 0.05 \%)$ and macrophages $(5.12 \pm 0.12 \%)$ significantly increased. The intermediate substance had a shallow 
granular appearance in the absence of necrotic masses and insignificant quantities of microflora. In the comparison group at the 5th day of treatment, the cytological picture was characterized by slow clearance from the microflora, polymorphonuclear leukocytes $70.03 \pm 1.58 \%$, of which degenerative forms were $68.76 \pm 1.68 \%$, monocytes $9.84 \pm$ $0.24 \%$, macrophages $2,43 \pm 0,06 \%$, fibroblasts $1,88 \pm 0,05 \%$, lymphocytes $15,82 \pm 0,38 \%$, phagocytosis $19,51 \pm 0,48 \%$, elements of fatty detritus, necrotic tissue.

The results of 100 microbiological studies of 74 patients with purulent wounds - the main group (36 patients) and the comparison group (38 patients) - have been analyzed. Most of the microbiological samples obtained in which the microflora was detected were monocultures: 20 patients $(55.6 \%)$ of the main group; in the comparison group, this figure was 30 patients (78.9\%). Two or more microorganisms were implanted in 16 patients (44.4\%) of the main group; in the comparison group, this figure was 8 patients (21.1\%). Gram-positive microflora was sown in $22(42.31 \%)$ samples of the main and 26 (56.52\%) of the comparison group. Gram-negative microflora was obtained in 30 samples $(57.69 \%)$ of the primary and 19 (41.30\%) of the comparison group. In one patient of the control group (2.18\%) yeast-like fungi of the genus Candida were obtained. The gram-positive flora of patients in the main group was presented in 20 (90.91\%) cases of staphylococci, among which Staphylococcus aureus was detected in 16 samples (80\% of all staphylococci) and Staphylococcus haemolitycus in 4 cases (20\% of all staphylococci). Other cocci were detected in $2(9,09 \%)$ cases. The gram-positive microflora of the comparison group in 21 cases $(80.78 \%)$ was represented by staphylococci, among which strains of Staphylococcus aureus were 18 cases ( $85.71 \%$ of all staphylococci), Staphylococcus haemolyticus was in 3 cases (14.29\% of all staphylococci) Enterococci were obtained in 3 cases (11.53\%), other Gram-positive microorganisms in 2 cases $(7.69 \%)$. The gram-negative flora of the main group was represented by the Enterobacteriaceae family $22(73.33 \%)$ cases of Acinetobacter (Moraxellaceae family) in 6 (20\%) cases and in 2 (6.67\%) cases of Pseudomonas aeruginosa species

The gram-negative microflora of the comparison group was presented by the Enterobacteriaceae family in 13 cases $(68.42 \%)$ and the genus Acinetobacter (Moraxellaceae family) in 6 cases $(31.58 \%)$. While analyzing the results of microbiological studies in patients with two or more microorganisms sown, the following pattern has been observed: in patients with the main group: mixed Gram-positive and Gram-negative flora found in 10 cases $(62.5 \%)$; only Gram-positive in 2 patients (12.5\%); only Gram-negative in 4 cases $(25.0 \%)$. In patients of the comparison group: mixed Gram-positive and Gram-negative flora found in 5 patients (62.5\%); only Gram-positive in 2 patients $(25.0 \%)$; only Gram-negative in 1 cases (12.5\%).

To assess antibacterial effectiveness, the following qualitative and quantitative indicators of microbiological study have been used: the number of colony-forming units (COCs) before, and after treatment after 5 and 10 days. The number of CSOs have also been taken into account when the replacement of the sowed microorganism took place; in polycultural crops, the number of CSFs of each microorganism has been evaluated separately; change in the species composition of the microflora; change in the sensitivity of microflora to antibacterial drugs in microbiological samples, in which during the treatment no specific changes in the microorganism occurred.

In the main group, after 5 days of treatment, a decrease in CSF after treatment was observed in 32 (76.19\%) cases, and it was expressed to varying degrees - the number of CVDs decreased by 100-1000 times (from $1 \times$ to $1 \times 10^{3}$, on average $\left.1.95 \times 10^{3}\right)$, in $7(16.67 \%)$ cases, the microbial growth rates remained the same, in 3 cases $(7.14 \%)$ the growth of CSF was observed in the range from $1 \times 10^{2}$ to $1 \times 10^{3}$.

At the same time, after 5 days of treatment in the comparason group, the following trend has been noted: the decrease in CSF in $24(54.55 \%)$ cases, the number of CVD has been observed in $12(27.27 \%)$ cases, the increase in CSO in $8(18.18 \%)$ cases.

After 10 days of treatment in the samples of the patients in the main group, the following results have been observed: the reduction of the CVS by an average of $1.2^{\wedge} 2$ occurred in $32(82.05 \%)$ cases, in $4(10.26 \%)$ cases, the number of CVS remained stable and in $3(7.69 \%)$ cases were marked by the growth of CSO. It should be noted that the species change of the pathogen in this case has not been taken into account.

In the comparison group, the average reduction in the number of CSOs was on average $1.44 \times 10^{3}$ and has been noted in 27 cases $(62.79 \%)$, without changes in the number - in 11 cases $(25.58 \%)$, in 5 cases (11.63\%), there was an increase in the number of CSOs. It should be noted that the evaluation of the results showed that the largest changes in the number of microorganisms in the patients in the main group occurred after 5 days of treatment, in contrast to the comparison group, in which similar indicators of microbial contamination remained high.

Regarding the species composition of the main group, the most expressed changes were noted in patients, the wound microflora of which was represented by two and more microorganisms - polycultural baccid. During treatment of 13 patients ( $81.25 \%$ of all patients with more than one microorganism sown), the monoculture of the pathogen was sown in repeated bacteriological studies of wound healing. In 7 (17.95\%) cases of all studies, replacement of the dominant pathogen with representatives of nosocomial infections - the Proteus group, Acinetobacter has been shown.

At the same time, in patients from a comparison group in which several pathogens were sown, significant differences have been noted, mainly in 5 cases $(62.5 \%)$ the species composition of the microflora remained the same, that is, monoculture after the course of treatment was sown only in 3 models (37.5\% of cases). The change in the pathogen in the comparison group was $11(25.58 \%)$ cases, and has been presented by the nosocomial microflora.

The dynamics of changes in the sensitivity to antibacterial agents of microbiological samples of patients in the main group after treatment for 10 days, which did not change 
Table I. Signs of inflammatory process of wounds in groups.

\begin{tabular}{|c|c|c|c|c|c|c|c|c|c|c|c|c|}
\hline \multirow{2}{*}{$\begin{array}{c}\text { Group } \\
\text { (main, comparison) }\end{array}$} & \multicolumn{4}{|c|}{ Hyperemia } & \multicolumn{4}{|c|}{ Edema } & \multicolumn{4}{|c|}{ Discharge } \\
\hline & 1 day & 3 day & 5 day & 7 day & 1 day & 3 day & 5 day & 7 day & 1 day & 3 day & 5 day & 7 day \\
\hline Main group $n=40$ & 2,24 & 1,32 & 0,76 & 0,36 & 2,4 & 1,4 & 0,68 & 0,28 & 2,2 & 1,2 & 0,64 & 0,32 \\
\hline Comparison group $n=45$ & 2,28 & 1,52 & 1,08 & 0,36 & 2,44 & 1,92 & 1,32 & 0,68 & 2,24 & 1,72 & 1,12 & 0,56 \\
\hline Indicator difference & 0,04 & 0,2 & 0,32 & 0 & 0,04 & 0,52 & 0,64 & 0,4 & 0,04 & 0,52 & 0,48 & 0,24 \\
\hline
\end{tabular}

Table II. Signs of wound healing in groups.

\begin{tabular}{|c|c|c|c|c|c|c|c|c|}
\hline \multirow{2}{*}{$\begin{array}{c}\text { Group } \\
\text { (main, comparison) }\end{array}$} & \multicolumn{4}{|c|}{ Granulation } & \multicolumn{4}{|c|}{ Epithelization } \\
\hline & 1 day & 3 day & 5 day & 7 day & 1 day & 3 day & 5 day & 7 day \\
\hline Main group, $\mathrm{n}=40$ & 0,32 & 1,12 & 1,8 & 2,08 & 0,08 & 0,2 & 0,88 & 1,56 \\
\hline Comparison group, $n=45$ & 0,28 & 0,76 & 1,36 & 1,8 & 0,04 & 0,2 & 0,92 & 1,28 \\
\hline Indicator difference & 0,04 & 0,36 & 0,44 & 0,28 & 0,04 & 0 & 0,04 & 0,28 \\
\hline
\end{tabular}

the species composition of the microflora (32 models), to some extent, correlated with the amount of CSF. So, in $21(65.63 \%)$ cases it did not change, in $6(18.75 \%)$ there was a sensitivity increase to some (mainly carbapenems) with a simultaneous decrease in the sensitivity to other antibacterial drugs. At the same time, in $5(15.62 \%)$ cases, an increase in resistance to antibacterial agents of the microflora has been observed.

In the comparative group in similar cases, when the species changes of the pathogen (32 cases) have not been observed, the picture was as follows: $17(53.13 \%)$ cases of sensitivity to antibacterial drugs did not appear, in $3(9.38 \%)$ cases there was a multidirectional change in sensitivity to antibacterial agents. At the same time, in $12(37.5 \%)$ of repeated microbiological studies, there was the development of low-sensitivity and resistant strains to certain types of antibacterial drugs.

In order to evaluate and compare the results of the study in patients with the main and the control group, clinical signs such as edema, hyperemia, wound secretions, the presence of granulation and wound epithelization and \pm signs were taken, each of the signs "+" was taken as a unit, and the sign " - "was zero. In this way, the ranking and detection of a significant difference in the parameters of the groups by statistical processing using the Wilcoxon $\mathrm{T}$ criterion $(p<0.05)$ has been performed. Subsequently, the analysis with the definition of the difference of indicators is carried out, see. tables I, II.

\section{DISCUSSION}

Undeniable is the fact that excessive microbial infestation continues the time of inflammation of the wound and slows healing. The use of antiseptics has a number of limitations, among which the greatest role is their cytotoxicity, especially in relation to proliferation and migration of fibroblasts [8]. Therefore, the local application of an antibiotic or a combination of them that acts selectively on the pathogen is an alternative solution, and the risk of developing resistance is significantly reduced if it is not used systematically. An essential advantage of antiseptics against antibiotics is the presence of a large number of dosage forms [9]. Aeration of the wound provides a wound with sufficient amounts of oxygen, especially under high pressure, has a detrimental effect on a number of microorganisms, primarily anaerobes, which in turn enhances the protective ability of the body [10]. The modern principle of wound treatment is the observance of the wet environment in the wound for mitosis, accelerating the growth of fibroblasts, the formation of fibronectin, the synthesis of vascular factor, maturation of granulation tissue. In addition, the moist environment provides high activity of wound proteases, prevents drying of nerve endings, and reduces pain syndrome [11]. It also reduces inflammatory reaction and scarring [12, 13]. Measurement and correction of the hydrogen index $(\mathrm{pH})$ of the wound has a diagnostic and therapeutic value for healing. $\mathrm{pH}$ affects the course of immune responses, the activity of proteases, including bacterial, release of oxyhemoglobin, the degree of neutropotic apoptosis, the expression of genes of pathogenic microorganisms, the production of biofilms and the effect on other components of microorganism virulence. Most antibacterial drugs for local treatment of purulent wounds are directly dependent on $\mathrm{pH}$. This is due to the change in the chemical composition of the drug and the change in the sensitivity of the microorganism [14]. Alkaline environment is a necessary condition for bacterial proteases, under such conditions, the synthesis of biofilms is risen [15]. The developed treatment technique combines such important elements for healing as wound drainage, microbial stress reduction, increased aeration and moisturizing of the wound, local application of antibiotic therapy in the form of an aerosol dispenser (aerosol), correction of the $\mathrm{pH}$ of the wound surface. The performed studies proved the effectiveness of the suggested treatment method, which was manifested in the rapid purification of wounds from the microflora and necrotic tissues and reduced amounts of degenerative forms of neutrophils already for 5 days in the patients of the main group compared with patients in the comparison group. In the main group, there was also a quick activation of the phagocytic response and regenerative capabilities of the organism, which was manifested in the emergence 
of macrophages and their precursors - monocytes. This indicates an acceleration of the inflammatory response of patients in the main group and a decrease in the duration of phase I of the wound process. In the analysis of microbiological studies, in patients with the main group, there was a decrease in the quantity of microorganisms in the wound in comparison with the control group in quantitative and qualitative terms, especially after the first 5 days of treatment. Indicators of microbial contamination remained higher in the comparison group even after 10 days of treatment compared with the main group. In the vast majority of patients in the main group there were minor changes in the sensitivity of the microflora that was sown at the beginning of treatment with antibacterial drugs, compared with the control group, which showed a high percentage of development of resistant strains. As a result, the use of the suggested integrated approach reduces the microbial burden on the wound, accelerates its purging from purulent-necrotic tissues and reduces the duration of the inflammatory process due to the presence of the microbial factor. Analyzing the clinical changes in the wounds of patients in the main group, it should be noted that they had a linear, declining nature, it was clinically characterized by a decrease in inflammatory changes in the wound, already after the first 3 days of treatment according to the method. In the comparative analysis it should be noted that the largest changes, the difference in the indicators of patients in the main group occurred in the indicators "Edema" and "Selection", somewhat less dynamics of changes was observed in the indicator "Hyperemia".

The analysis of the obtained data showed a positive effect of this type of treatment method on the early appearance of granulation tissue, and its lesser effect on the epithelization process compared with the control group. In each particular case, the presence of concomitant vascular pathology and metabolic changes should be taken into account, but the tendency of the healing process indicates a clear positive effect of this method of treatment on wound healing and the appearance of granulations earlier in patients with the main group compared to the control group.

\section{CONCLUSIONS}

The suggested treatment method has several advantages and allows: to quickly achieve a therapeutic effect, especially pain relieving when using local anesthetics; to increase the efficiency of the medicinal product, due to even distribution throughout the wound surface; to reduce the toxic effect of the medicinal product on wound tissue present in the case of the use of concentrated antiseptic agents; to treat in a natural, moist environment; step by step use of different drugs without mixing, which reduces the possibility of their mutual chemical inactivation; to reduce wound cell hypoxia by its aeration; to improve drainage efficiency, since excess pressure pushes out the exudate and reveals the closed wound areas in hard-to-reach areas. The main clinical features that characterized and distinguished the main group from the comparison group were the faster cleansing of the wound, respectively, the faster reduction of edema and secretions in this group, and as a consequence of the early appearance of granulation tissue. There are still unresolved questions about the application of the method on anatomical sites, where it is technically difficult to create a sealed space above the wound, there is a need to further study the effects of local antibiotic therapy under high pressure on bio pellicle formed by several types of microorganisms, the depth of penetration of antibacterial drug in the tissue, optimal dimension of pressure of the aerosol dispenser mixture.

\section{REFERENCES}

1. Dreifke M.B., Jayasuriya A.A., Jayasuriya A.C. Current wound healing procedures and potential care. Mater Sci Eng C Mater Biol Appl. 2015;48:651-662.

2. Sartelli M., Guirao X., Hardcastle T.C. et al. 2018 WSES/SIS-E consensus conference: recommendations for the management of skin and softtissue infections. World Journal of Emergency Surgery. 2018;13:58.

3. Sartelli M., Malangoni M.A., May A.K. et al. World Society of Emergency Surgery (WSES) guidelines for management of skin and soft tissue infections. World J Emerg Surg. 2014;9:57.

4. Andriushchenko V.P., Vashchuk V.V., Herych H.I. et al. Infektsii shkiry i miakykh tkanyn: suchasni pohliady i stratehiia antybiotykoterapii [Infections of skin and soft tissues: modern views and strategies of antibiotic therapy]. Health of Ukraine: Surgery. Orthopedics. Traumatology: thematic number. 2017; 2:12-14. (UA)

5. Agren M.S., Ostenfeld U., Kallehave F. et al. A randomized, double-blind, placebo-controlled multicenter trial evaluating topical zinc oxide for acute open wounds following pilonidal disease excision. Wound Repair and Regeneration. 2006;15:526-535.

6. Norman G., Dumville J.C., Mohapatra D.P. et al. Antibiotics and antiseptics for surgical wounds healing by secondary intention. Cochrane Database of Syst Rev. 2016;3:711-712.

7. Smyth E.T., Mcllvenny G., Enstone J.E. et al. Four country healthcare associated infection prevalence survey 2006: overview of the results. Journal of Hospital Infection. 2008;69(3):230-248.

8. Cooper M. The cytotoxic effects of commonly used topical antibacterial agents on human fibroblasts and keratinocytes. J Trauma. 1991;1:782-784.

9. Allen D., Maguire J., Mahdavian M. et al. Wound hypoxia and acidosis limit neutrophil bacterial killing mechanisms. Arch Surg. 1997;132:991-996.

10. Yamane T., Nakagami G., Yoshino S. et al. Hydrocellular Foam Dressing Promotes Wound Healing along with Increases in Hyaluronan Synthase 3 and PPARa. Gene Expression in Epidermis. PLOS ONE. 2013;8:8.

11. Junker J.P., Kamel R.A., Caterson E.J. et al. Clinical impact upon wound healing and inflammation in moist, wet, and dry environments. Advances In Wound Care. 2013;2(7):348-354.

12. Shaprynskyi V.0., Shaprinskiy Y.V., Karyi Y.V. et al. Operative treatment of corrosive esophageal strictures. Wiadomości Lekarskie. 2018;71(2):323-325.

13. Percival S.L., McCarty S., Hunt J.A. et al. The effects of $\mathrm{pH}$ on wound healing, biofilms, and antimicrobial efficacy. Wound Repair and Regeneration. 2014;22:174-186.

14. Emrich N., Heisig A., Stubbings W. et al. Antibacterial activity of finafloxacin under different $\mathrm{pH}$ conditions against isogenic strains of Escherichia coli expressing combinations of defined mechanisms of fluoroquinolone resistance. Journal of Antimicrobial Chemotherapy. 2010;65:2530-2533. 
15. Greener B., Hughes A.A., Bannister N.P. et al. Proteases and pH in chronic wounds. J Wound Care. 2005;14:59-61.

The article is a part of complex scientific research work at Surgery Department of National Pirogov Memorial Medical University, Vinnytsya, "Development and improvement of advanced technologies in surgical treatment and prevention of postoperative complications in patients with diseases of abdominal and chest cavities", state registration number 0113 U007692.

\section{ORCID and contributionship:}

Volodymyr O. Shaprynskyi - 0000-0002-3890-6217 A,B

Stepan S. Skalskyi - 0000-0002-3351-5399 B,C,D

Yevhen V. Shaprynskyi - 0000-0002-4949-3163 ${ }^{D, E, F}$

Andriy V. Verba - 0000-0002-9661-3084 ${ }^{B, F}$

Viktor M. Makarov - 0000-0003-2100-1398 ${ }^{B, F}$

\section{Conflict of interest:}

The Authors declare no conflict of interest.

\section{CORRESPONDING AUTHOR} Volodymyr 0 . Shaprynskyi

National Pirogov Memorial Medical University,

Pirogov street, 56, 21018, Vinnytsia, Ukraine

tel: +380952190209

e-mail: skstst40@gmail.com

Received: 29.01.2020

Accepted: 31.03 .2020

A - Work concept and design, B - Data collection and analysis, C - Responsibility for statistical analysis,

D-Writing the article, $\mathbf{E}$-Critical review, $\mathbf{F}$ - Final approval of the article 